\title{
Dosage immunoradiométrique de la rénine active et de la prorénine plasmatiques au cours des cycles stimulés pour FIVETE ou GIFT
}

\author{
Arlette GUICHARD, Thérèse-Marie MIGNOT, P. BOYER, J. R. ZORN, Lise \\ CEDARD
}

Centre de Fécondation In Vitro Baudelocque - Saint-Vincent-de-Paul et $U .166$ INSERM 123, bd de Port-Royal, 75014 Paris.

Summary. Immunoreactive renin variations as a predictive index of the outcome after in vitro fertilization and embryo transfert or intrafallopian gamete transfer.

Prorenin (PR) an inactive high molecular weight form of renin normally circulates in human plasma at a concentration of about 10 times that of active renin (AR) and this proenzyme seems to be linked to the reproductive function. It has been demonstrated that $A R$ and $P R$ are present at high concentrations in follicular fluid when the ovaries are stimulated with gonadotropins and that the PR plasma levels increase steadily after hCG injection with a correlation between blood PR and the number of developing follicles and corpora lutea. From September 1986 we studied the profile of immunoreactive active renin (AR) and prorenin (PR) in plasma during hyperstimulated cycles for IVFET or GIFT. All women were treated with a protocol combining $\mathrm{GnRH}$ analog (Decaptyl Ipsen Biotech, Paris France) and human menopausal gonadotropins until injection of 5000 IU hCG. AR has been assayed in frozen samples by specific immunoradiometry (Renin RIA code 79 795, Pasteur Diagnostic, France) using two complementary monoclonal antibodies. A second assay of total renin was carried out after trypsin activation which revealed the inactive form. Progesterone $(P)$, estradiol $\left(E_{2}\right)$ were measured by radioimmuno-assays. During the follicular phase, from day 1 of $\mathrm{hMG}$ administration to the day before hCG, no significant difference could be found between two groups, 63 pregnant or 60 nonpregnant cycles matched for age and number of oocytes retrieved, for $E_{2}, P, P R$ and $A R$. During the periovulatory period ( $D-1, D_{0}=$ day of $h C G$ injection and $D 1$ ) no difference could be found for $E_{2}, P$ and $P R$ (tabl. 1). In the 2 groups the mean $E_{2}$ levels increased after hCG injection, as well as P and PR. But a significant difference appeared for AR which increased in the plasma immediately after hCG administration in the pregnant group whereas it decreased in the non-pregnant group $(+2,5 \mathrm{vs}-2 \mathrm{pg} / \mathrm{ml})$ the mean variation between $D_{O}$ and $D+1$ being significantly different in fertile cycles and in nonfertile cycles. We could thus distinguish a population with a high probability of prenancy (fig. 3$)(71 \%$ with $D_{O}-D 1$ increase $>4 \mathrm{pg} / \mathrm{ml}$, and a low probability $(21 \%$ in case of a significant decrease). During the luteal phase P, PR and AR increased in all the women (fig. 4), but returned to basal levels in the absence of pregnancy, whereas they increased during the late luteal phase in fertile cycles when hCG began to be secreted and their variations in the same patients had a predictive value of pregnancy as early as $D_{9}-D_{10}(56 \pm 12 \mathrm{vs}$ $25 \pm 3 \mathrm{pg} / \mathrm{ml}) \mathrm{p}<0.02$

\section{Introduction.}

La rénine, aspartyl protéase, dérivée de la préprorénine après clivage successif d'un peptide signal puis d'une chaîne polypeptidique de 46 acides aminés est 
classiquement considérée comme une enzyme synthétisée, stockée et sécrétée par le rein. Il est généralement admis que les précurseurs de ce type sont transformés en hormone active au cours de leur stockage intracellulaire ou de leur libération dans la circulation. La rénine est I'enzyme limitante d'une cascade de réactions biochimiques qui catalysent la transformation de l'angiotensinogène produit par le foie en angiotensine I et angiotensine II puissant agent vaso-constricteur, impliqué dans la régulation de la pression artérielle (1).

La rénine est présente dans la circulation sanguine sous deux formes: la prorénine inactive et la rénine biologiquement active qui ne représente que $10 \%$ de la précédente (2) et disparaît du plasma après néphrectomie bilatérale, alors que $10 \%$ de la prorénine est d'origine extra-rénale, et persiste dans le sang après néphrectomie (3-4). En effet, de nombreux organes synthétisent la prorénine tels que le placenta, l'utérus, l'ovaire, les surrénales, I'hypophyse antérieure et le testicule, mais tous ne sécrètent pas de la rénine active dans la circulation générale (5). L'expression du gène de la rénine dans les tissus extra-rénaux est soumise à des influences hormonales diverses selon les espèces et variables selon le stade de développement, la sécrétion de prorénine par les gonades étant plus particulièrement stimulée par les hormones gonadotropes. Dans l'espèce humaine, l'existence d'une sécrétion ovarienne de prorénine est maintenant bien démontrée et ses variations plasmatiques au cours des cycles spontanés ou stimulés ont été récemment décrites (6).

Il a également été montré que le liquide folliculaire prélevé lors des ponctions ovocytaires est très riche en prorénine et qu'il contient aussi de la rénine active et de l'angiotensine (7-8). Au cours du cycle menstruel, la prorénine, faible en phase folliculaire, augmente après le pic de LH et revient aux taux de base en milieu de phase lutéale, la rénine active variant très peu (6).

Au cours des cycles stimulés par clomiphène ou hMG, la prorénine plasmatique augmente rapidement après l'administration d'hCG (8-9-10-11), en corrélation avec le nombre de follicules et de corps jaunes (10-11). La fécondation in vitro avec transfert d'embryon (FIVETE) et le transfert intra-tubaire des gamètes (GIFT) nous ont paru des modèles privilégiés pour l'étude des facteurs stimulant la synthèse de prorénine et nous avons plus particulièrement recherché l'existence d'une différence dans les taux de rénine active et de prorénine plasmatiques entre les cycles fertiles et infertiles et étudié leur corrélation avec la progestérone, l'œstradiol et l'hCG (12).

\section{Matériel et méthodes.}

Nous avons étudié par immunoradiométrie le profil plasmatique de la rénine et de la prorénine dans 123 cycles hyperstimulés pour FIVETE ou GIFT chez 112 femmes de 23 à 41 ans. 69 FIVETE ont été effectuées pour cause de stérilité tubaire et 54 GIFT essentiellement pour stérilité idiopathique. 63 cycles fertiles après transfert embryonnaire ou GIFT (groupe 1) ont été rétrospectivement comparés à 60 cycles non fertiles (groupe 2) après avoir été appariés en fonction de l'âge des patients ( $32 \pm 4,4$ et $33 \pm 4,1$ ) et du nombre d'ovocytes ponc- 
tionnés $(6,7 \pm 3,4$ et $6,6 \pm 3,4)$. Dans tous les cycles la stimulation ovarienne a été programmée par la séquence norethisterone-hMG combinée au D-Trp-6-LHRH (Decapeptyl: Ipsen Biotech. Paris, France), administré par voie intramusculaire (IM) ou sous-cutanée (SC), selon le protocole précédemment décrit (13). Lorsque l'œstradiol a atteint un taux $\geqslant 1100 \mathrm{pg} / \mathrm{ml}$ en accord avec la présence de plusieurs grands follicules (diamètre $>15 \mathrm{~mm}$ ) une injection de 5000 UI d'hormone gonadotrope chorionique (hCG) est effectuée le soir même (J.0.). A partir du $5^{\mathrm{e}}$ jour de l'administration d'hMG jusqu'à celui de la ponction ovocytaire les prises de sang sont effectuées quotidiennement à $8 \mathrm{~h}$ du matin, sur héparine (150 UI) pour le dosage rapide d'œestradiol, et des échantillons sont immédiatement stockés à $-20^{\circ}$. Progestérone $(P)$, rénine active $(R A)$ et prorénine $(P R)$ sont dosées ultérieurement après une seule décongélation.

Pendant la phase lutéale trois prises de sang au minimum ont été réalisées avant le premier dosage de l'hCG effectué environ 11 jours après le transfert. La rénine active a été dosée par immunoradiométrie (Renin RIA code 79795 , Pasteur Diagnostic, Marnes-la-Coquette, France) à l'aide de 2 anticorps monoclonaux complémentaires (14). Le premier anticorps monoclonal 3E8 est fixé de façon covalente sur une phase solide magnétique, le second $4 \mathrm{G} 1$ spécifique de la rénine active est marqué à l'iode 125 . Un second dosage de la rénine totale est effectué après trypsinisation qui active la prorénine. La concentration en prorénine est calculée par la différence entre la rénine totale et la rénine active. $\mathrm{La}$ sensibilité du dosage est de $2 \mathrm{pg} / \mathrm{ml}$. La reproductibilité intra-dosage pour 15,5, 35,5 et $298 \mathrm{pg} / \mathrm{ml}$ de rénine plasmatique est respectivement de $11,8,5,7$ et $1,7 \%$, la reproductibilité inter-dosage de 16,8, 10,8 et $6 \%$.

L'œstradiol et la progestérone ont été dosés par radioimmunologie avec des trousses du commerce (ER : EIR 150 Wurenligen, Suisse, ${ }^{125}$ Progesterone CoA Tria 66380 - Biomérieux, Marcy-l'Etoile, France). L'étude statistique a été effectuée par l'analyse du chi 2 et par le test $t$ de Student sur des échantillons appariés.

\section{Résultats.}

Les résultats concernent une étude rétrospective effectuée sur des plasmas congelés à $-20^{\circ} \mathrm{C}$ et décongelés une seule fois. Pendant la phase folliculaire, au cours de la stimulation par hMG jusqu'au jour précédant l'injection de hCG $(\mathrm{J}-1)$, il n'y a aucune différence significative entre les deux groupes de femmes enceintes et non enceintes, pour $E_{2}, P, P R$ et $A R$ (fig. 1). Pendant la phase périovulatoire $J 0$ et $J+1$ ) aucune différence n'apparaît pour $E_{2}, P$ et $P R$. Dans les deux groupes de femmes, les taux moyens de $E_{2}$ augmentent après l'injection de hCG ainsi que ceux de P et PR (tabl. 1). Par contre une différence significative apparaît pour la rénine active, qui augmente dans le plasma immédiatement après l'administration d'hCG dans le groupe des femmes enceintes (FIV $\mathrm{n}=36$, GIFT $\mathrm{n}=27$ ) alors qu'elle diminue transitoirement dans l'autre groupe (fig. 2). Les variations entre $\mathrm{J} 0$ et $\mathrm{J}+1$ sont significativement différentes, leurs moyennes étant supérieures au niveau de base $(+2,5 \mathrm{pg} / \mathrm{ml})$ dans les cycles fertiles et 
inférieurs $(-2 \mathrm{pg} / \mathrm{ml})$ dans les cycles non fertiles $(P<0,001)$. La figure 3 montre la répartition des grossesses en fonction de la réponse spécifique de la rénine active à l'injection déclenchante de hCG. Dans les 35 cycles pour lesquels la RA augmente entre $\mathrm{J} 0$ et $\mathrm{J}+1$ de plus de $4 \mathrm{pg} / \mathrm{ml}$ nous avons retrouvé 25 grossesses soit un taux de $71 \%$ par cycle. Dans le cas où la RA est restée stationnaire nous avons obtenu 33 grossesses sur 64 cycles soit $52 \%$. Enfin dans 24 cycles où la RA a chuté nous avons eu seulement 5 grossesses soit $21 \%$. Le fort pourcentage de grossesses obtenues dans le groupe avec augmentation immédiate de la RA après hCG est similaire dans les cycles de FIV et ceux de GIFT $(67 \%$ et $76 \%)$.
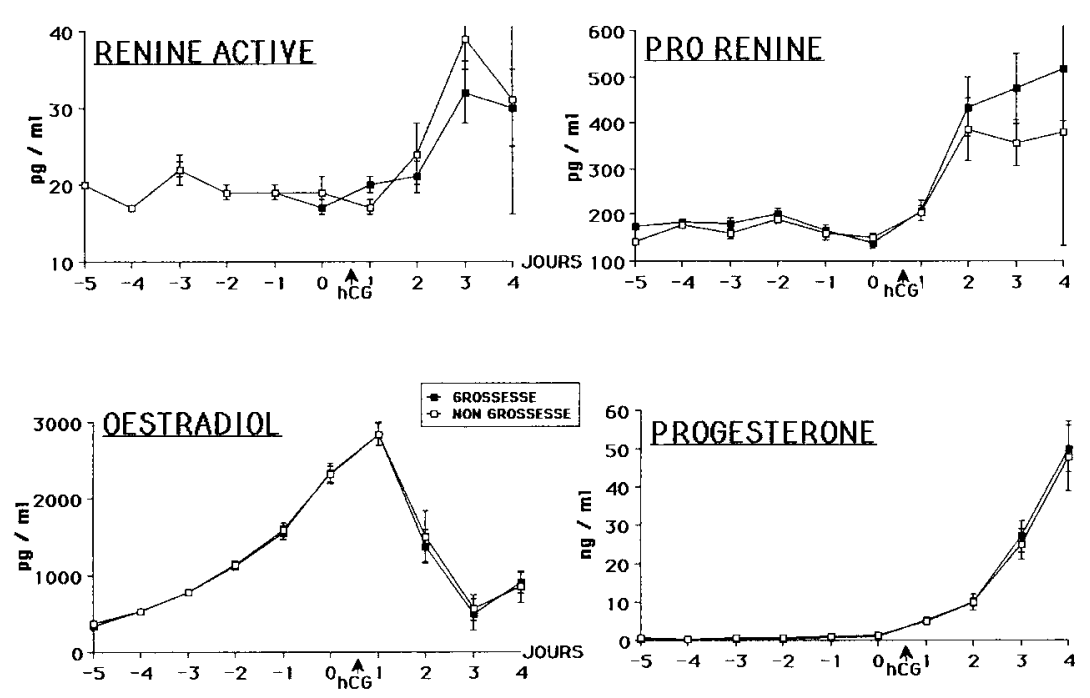

FIG. 1. - Valeurs moyennes ( \pm écart type de la moyenne) de l'oestradiol, de la progestérone, de la prorénine et de la rénine active dans le plasma au cours de la stimulation ovarienne pour F/V ou GIFT mesurées dans 63 cycles fertiles et dans 60 cycles infertiles.

L'analyse statistique par le test chi 2 a établi que l'hétérogénéité des résultats est globalement significative $(P<0,001)$ et que la probabilité d'une grossesse est significativement plus faible en cas de diminution de la rénine active entre $\mathrm{J} 0$ et $J+1$. II n'y a aucune différence significative entre les groupes stationnaire et ascendant ni entre FIVETE et GIFT.

Durant la phase lutéale précoce (fig. 4), P, PR et RA augmentent chez toutes les femmes. On observe un pic de PR entre $J 2$ et $J 4$, la concentration de cette proenzyme étant corrélée au nombre d'ovocytes ponctionnés chez les femmes enceintes ou non enceintes. II existe également une corrélation linéaire entre PR et $P(n=0,75, p<0,001)$. La $P R$ décroît ensuite, pour retourner à son niveau de base en absence de grossesse; en cas de cycle fertile elle augmente en 

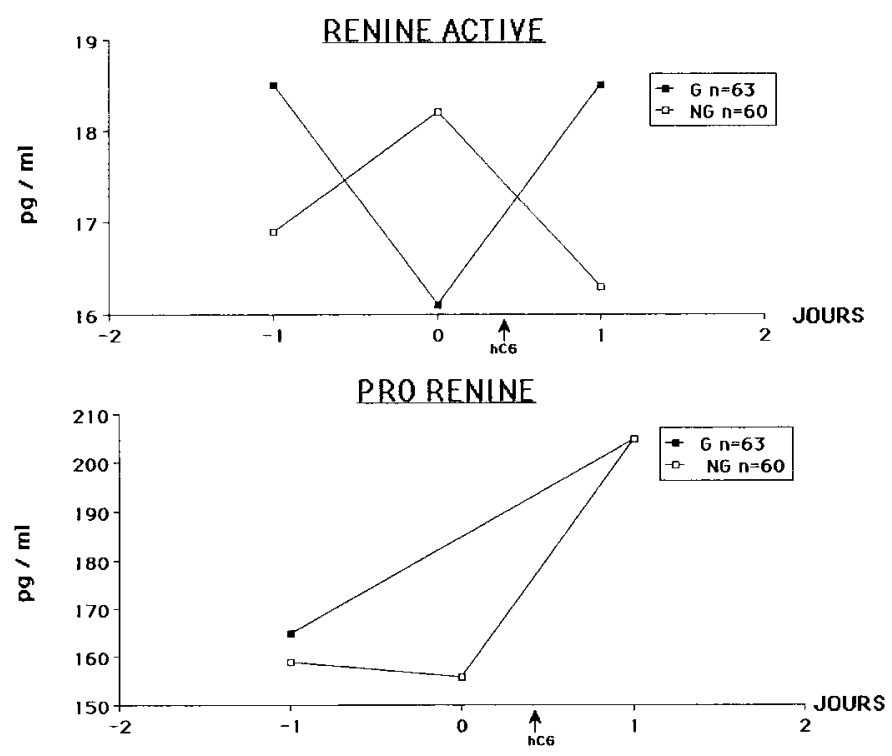

FIG. 2. - Variations de la rénine active et de la prorénine plasmatiques en période périovulatoire dans 63 cycles fertiles et 60 cycles infertiles. 500 UI d'hCG sont injectées le jour 0 à $22 \mathrm{~h}$.

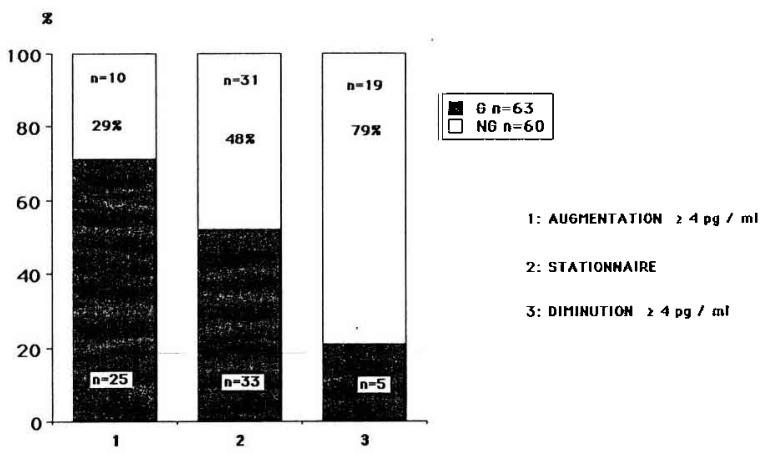

FIG. 3. - Distribution des grossesses selon le type de variations de la rénine active dans le plasma après injection d'hCG.

deuxième partie de la phase lutéale, la différence entre les deux groupes devenant hautement significative à $J 15(P<0,001)$, soit 11 jours après le transfert embryonnaire. Ce profil est comparable à celui de la progestérone qui augmente en début de phase lutéale, décroît transitoirement à $\mathrm{J} 10$ puis s'élève à nouveau lorsqu'il y a grossesse. Dans ce cas, la RA augmente également en milieu de phase lutéale ; la différence entre les deux groupes devient significative dès J $9-\mathrm{J} 10$ $(56 \pm 12$ vs $25 \pm 3 \mathrm{pg} / \mathrm{ml}) \mathrm{P}<0,02$. 


\section{TABLEAU 1}

Valeurs moyennes \pm écart type de la moyenne de la rénine active (RA), de la prorénine (PR), de l'cestradiol $\left(\mathrm{E}_{2}\right)$ et de la progestérone $(\mathrm{P})$ dans le plasma au cours de cycles fertiles ou non fertiles après transfert d'embryons ou de gamètes appariés en fonction de l'áge et du nombre d'ovocytes.

\begin{tabular}{|c|c|c|}
\hline & Grossesse $\mathrm{n}=63$ & Non-grossesse $n=60$ \\
\hline & FIV $n=36 \quad$ GIFT $n=27$ & FIV $n=33 \quad$ GIFT $n=27$ \\
\hline Ovocytes & $6,71 \pm 0,44$ & $6,60 \pm 0,44$ \\
\hline Age & $32 \pm 0,5$ & $33 \pm 0,5$ \\
\hline $\mathrm{RA} \mathrm{pg} / \mathrm{ml}$ & $\begin{array}{cc}J 0 & J+1 \\
16,1+1,3 & 18,5 \pm 1,3 \\
\end{array}$ & $\begin{array}{cc}J 0 & J+1 \\
18,2 \pm 1,5 & 16,3 \pm 1,3 \\
\end{array}$ \\
\hline PR pg/ml & $\begin{array}{c}J 0 \\
167+12,8 \\
\end{array}$ & $\begin{array}{c}J 0 \\
156 \pm 12,6\end{array}$ \\
\hline $\mathrm{E} 2 \mathrm{pg} / \mathrm{ml}$ & $\begin{array}{c}J+1 \\
2880 \pm 155\end{array}$ & $\begin{array}{c}\mathrm{J} 0 \\
2315+114 \\
\end{array}$ \\
\hline $\mathrm{P} 4 \mathrm{ng} / \mathrm{ml}$ & $\begin{array}{c}J+1 \\
5,3 \pm 0,6\end{array}$ & $\begin{array}{c}J+1 \\
4,9 \pm 0,5\end{array}$ \\
\hline
\end{tabular}

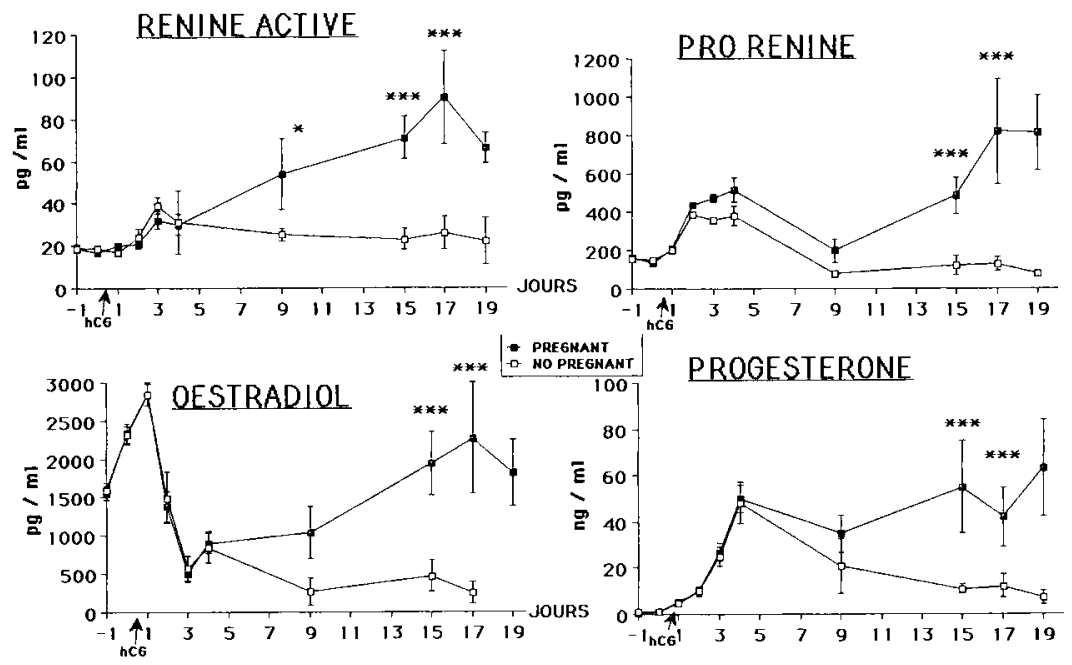

FIG. 4. - Valeurs movennes ( \pm écart type de la moyenne) de l'oestradiol, de la progestérone, de la rénine active et de la prorénine au cours de la période périovulatoire et de la phase lutéale chez 39 femmes enceintes et 35 femmes non enceintes.

La comparaison des valeurs de la rénine active dans le plasma au cours des diverses périodes du cycle chez la même femme permet d'éliminer les variations dues à des facteurs individuels (âge, type de stimulation, nombre de follicules...). La figure 5 représente le pourcentage de variation calculé d'après la différence entre les valeurs obtenues avant l'injection d'hCG ( $J 0)$ et le lendemain ( $\mathrm{J} 1$ ) et entre $\mathrm{J} 9$ et $\mathrm{J} 10$, divisé par la valeur de base à $\mathrm{J} 0$. On constate qu'il est plus élevé dans les cycles fertiles en période périovulatoire $(+9 \%)$ au lieu de $-8 \%$ ) et surtout en milieu de phase lutéale ( $+244 \% v s+45 \%)$. Il en est de même en fin 
de phase lutéale où la rénine active augmente entre $\mathrm{J} 9$ et $\mathrm{J} 15$ ( $+47 \%$ ) alors qu'elle diminue en cas d'échec d'implantation ( $-12 \%)$.

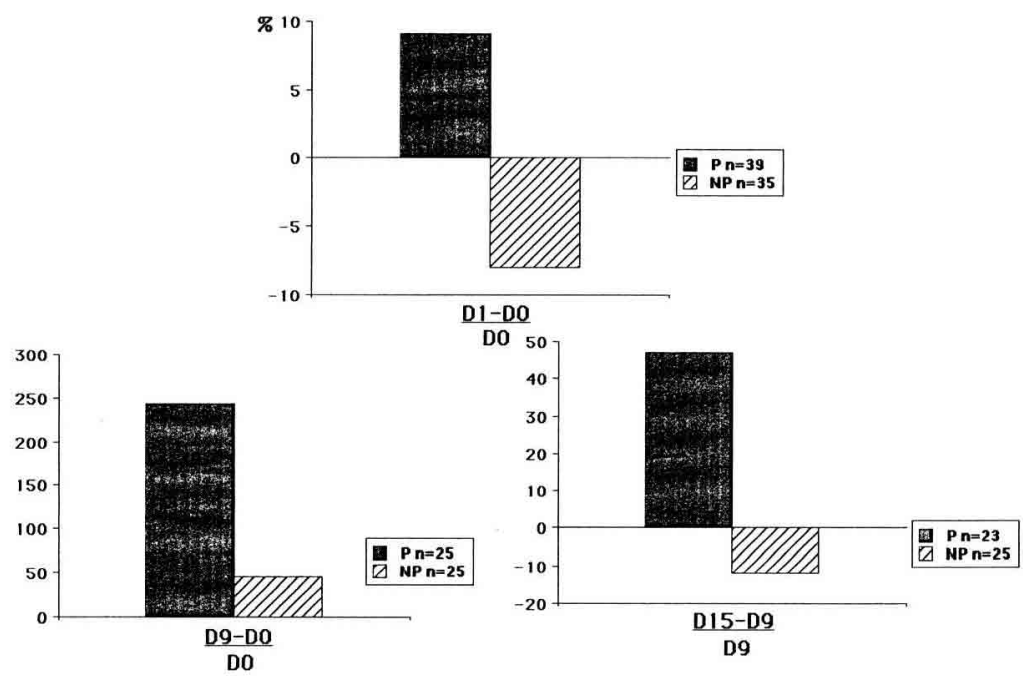

FIG. 5. - Variations des taux de rénine active plasmatique au cours des diverses périodes des cycles stimulés pour FIV ou GIFT des femmes enceintes (P) ou non enceintes (NP) exprimées en pourcentage par rapport aux taux de base ( $\mathrm{J} O$ pour la période périovulatoire et la phase lutéale précoce, $\mathrm{J} 9$ pour la phase tardive).

\section{Discussion.}

Les profils de la PR observés chez ces patients sont en accord avec les résultats récents de la littérature obtenus chez des femmes sous stimulation ovarienne par gonadotropines (9-10). Les taux plasmatiques de PR augmentent notablement après l'administration de hCG (7-8). Une corrélation positive entre la PR plasmatique, le nombre de follicules et des corps jaunes a récemment été observée (10-11) ainsi qu'entre $P R$ et $E_{2}$ ou $P$ pendant la phase lutéale, la prorénine augmentant rapidement en cas de grossesse, l'existence d'une corrélation hautement significative entre $P R$ et $P(15)$ suggère un facteur stimulant commun. Cependant la production de stéroïdes n'est pas directement responsable de cette stimulation; en effet les taux de PR sont faibles en cas de don d'embryons avec agénésie ovarienne et thérapie stéroïdienne de substitution (10-16).

Notre étude (11-12) est la première à rapporter une valeur prédictive du dosage de la rénine active plasmatique dont la réalisation est plus facile par immunoradiométrie que par la conversion enzymatique de l'angiotensinogène en angiotensine I.

Le dosage direct de la RA présente une meilleure sensibilité et permet de mesurer exactement le nombre de molécules de RA présentes dans le plasma, indépendamment de la concentration de prorénine inactive dans l'échantillon et des conditions in vitro du dosage enzymatique. 
II a été démontré avec cette même méthode que la concentration de RA est 10 fois supérieure dans le liquide folliculaire des femmes stimulées par gonadotropines à celle du plasma (9). Les quatre composants du système rénineangiotensine, c'est-à-dire PR, RA, substrat de la rénine et l'enzyme de conversion de l'angiotensine, sont présents dans le liquide folliculaire (16) sans qu'il existe de corrélation entre la concentration de la rénine et du substrat (17). Très récemment le site de production de la prorénine a été localisé dans les cellules de la thèque en culture avec libération de $10 \%$ de rénine active (18).

L'activation spontanée de la prorénine plasmatique par le froid a été très bien étudiée par Derkx (19) et pourrait éventuellement être à l'origine d'un artéfact dans nos mesures de rénine active dont l'origine ovarienne directe est contestée (5). Ceci est cependant improbable car tous les échantillons de plasma sont immédiatement congelés et ne sont décongelés qu'une seule fois. De plus le pourcentage de RA que nous avons constaté est de l'ordre de $10 \%$ de la rénine totale comme pour l'ensemble des auteurs. Par ailleurs nous avons pu observer successivement chez 5 patientes, pendant la période périovulatoire les deux différents profils de la RA entre $\mathrm{J} 0$ et $\mathrm{J}+1$ : cycle stimulé infertile avec RA descendante et cycle stimulé fertile avec RA ascendante ou stationnaire.

A la suite de ces résultats nous avons effectué une étude prospective des variations de la RA en période périovulatoire, pendant un mois sur tous les cycles stimulés (soit 70 cycles) sans appariement préalable en fonction de l'âge des patientes ou du nombre d'ovocytes ponctionnés. Ces résultats prospectifs mais obtenus après congélation des échantillons confirment les précédents. Dans les 13 cycles ayant eu une RA augmentée entre $\mathrm{J} 0$ et $\mathrm{J}+1 \mathrm{~d}$ 'au moins $4 \mathrm{pg} / \mathrm{ml}$, nous avons obtenu 5 grossesses ( $38 \%$ ). Avec une RA stationnaire (41 cas) nous avons obtenu 9 grossesses ( $22 \%$ ). Enfin dans les cycles avec diminution de la RA d'au moins $4 \mathrm{pg} / \mathrm{ml}$ nous n'avons obtenu aucune grossesse.

Cependant l'annulation de la ponction ovocytaire en cas de diminution de la $\mathrm{RA}>4 \mathrm{pg} / \mathrm{ml}$ entre $\mathrm{J} 0$ et $\mathrm{J}+1$ est difficilement envisageable actuellement.

En effet sur le plan pratique le dosage quotidien de la rénine active pour étudier ses variations après l'injection de hCG, pose un problème technique car le coefficient de variation inter-essais aux faibles valeurs est de 16,8\% c'est-à-dire supérieur au pourcentage de variation entre $\mathrm{J} 0$ et $\mathrm{J}+1$. De plus l'activation par le froid de la prorénine (19) empêche la comparaison d'échantillons frais ou conservés $24 \mathrm{~h}$ à $4{ }^{\circ} \mathrm{C}$ qui aurait permis d'effectuer les mesures à $\mathrm{J} 0$ et $\mathrm{J}+1$ dans une même série de dosages et cette activation par le froid n'est pas empêchée par l'addition d'inhibiteur de la trypsine.

Cependant les résultats que nous avons obtenus rétrospectivement nous ont permis d'identifier dans les cycles stimulés pour FIVET ou de GIFT une population à haute probabilité de grossesse (71\%) avec une augmentation de RA entre J 0 et $\mathrm{J}+1$ et une autre population à faible probabilité $(21 \%)$ en cas de diminution significative de la RA plasmatique.

Le mécanisme de variation de la RA en période périovulatoire est encore inconnu mais on peut penser que l'élévation plus rapide de la rénine active dans les cycles fertiles traduit soit la synthèse d'enzymes protéolytiques, soit une vascularisation plus rapide du follicule. Ces résultats confirment que le système 
rénine-angiotensine ovarien est bien impliqué dans la physiologie ovarienne et dans la qualité de l'ovulation.

La formation d'angiotensine peut également jouer un rôle dans la vascularisation de l'aire d'implantation du jeune embryon, et se traduirait par l'augmentation significative de la PR et de la RA plasmatiques en milieu de phase lutéale que nous avons observée en cas de grossesse débutante.

$27^{e}$ Réunion de la Société française pour l'Etude de la Fertilité. Paris, 29, 30 sept., $1^{\text {er }}$ oct. 1988.

Remerciements. - Nous remercions très vivement les Drs Ch. Brami, S. Epelboin, C. Nathan, P. Quantin, F. Rollet, M. Savale pour leur coopération et le $\mathrm{Pr} P$. Corvol et le $\mathrm{Dr}$ T. Guyenne pour leurs précieux conseils.

\section{Références}

(1) LARAGH J. H., SEALEY J. E., 1973. The renin-angiotensin-aldosterone hormonal system and regulation of sodium, potassium, and blood pressure homostasis, 831-908. In ORLOFF J., BERLINER R. W. Handbook of Physiology : renal physiology. Am. Physiol. Soc., Waverly Press, Baltimore.

(2) SEALEY J. E., ATLAS S. A., LARAGH J. H., 1980. Prorenin and other large molecular weight form of renin. Endocr. Rev., 1, 365-391.

(3) WEINBERGER M. H., WADE M. B., AOI W., USA T., DENTIMO M., LUFT F., GRIM C. E., 1977. An extrarenal source of « renin-like » activity in anephric man. Circ. Res. (suppl. 1), 40, $1-4$

(4) SEALEY J. E., WHITE R. P., LARAGH J. H., RUBIN A. L., 1977. Plasma prorenin and renin in anephric patients. Circ. Res. (suppl. 2), 41, 17-21.

(5) SEALEY J. E., GOLdSTEIN M., PITARRESI T., KUDlAK T. T., GLORIOSO N., FIAMENGO S. A., LARAGH J. H., 1988. Prorenin secretion from human testis: No evidence for secretion of active renin or angiotensinogen. J. clin. Endocr. Metab., 66, 974-977.

(6) SEALEY J. E., CHOLST I., GLORIOSO N., TROFFA C., WEINTRAUB I. D., JAMES G., LARAGH J. H., 1987. Sequential changes in plasma luteinizing hormone and plasma prorenin during the menstrual cycle. J. clin. Endocr. Metab., 65, 1-5.

(7) FERNANDEZ L. A., TARLATZIS B. C., RZASA P. J., CARIDE V. J., LAUFER N., NEGRO-VILAR A. F., DE CHERNEY A. H., NAFTOLIN F., 1985. Renin like activity in ovarian follicular fluid. Fert. Ster., 44, 219-223.

(8) GLORIOSO N., ATLAS S. A., LARAGH J. H., JEWELEWICZ R., SEALEY J. E., 1986. Prorenin in high concentrations in human ovarian follicular fluid. Science, 233, 1422-1424.

(9) DERKX F. H. M., ALBERDA A. T., ZEILMAKER G. H., SCHALEKAMP M. A. D., 1987. High concentrations of immunoreactive renin, prorenin and enzymatically active renin in human ovaries follicular fluid. Brit. J. Obst. Gynaecol., 94, 4-9.

(10) ITSKOVITZ J., SEALEY J. E., GLORIOSO N., ROSENWAKS Z., 1987. Plasma prorenin response to human chorionic gonadotropin response to human chorionic gonadotropin in ovarian-hyperstimulated women : correlation with the number of ovarian follicles and steroid hormone concentrations. Proc. nat. Acad. Sci. USA, 84, 7285-7289.

(11) CEDARD L., MIGNOT Th. M., GUICHARD A., BOYER P., ZORN J. R., 1987. Immunoreactive renin and prorenin plasma variations during the periovulatory period in conception and non conception cycles after in vitro fertilization and embryo transfer. Human Reprod., 2, 48.

(12) GUICHARD L., MIGNOT Th. M., BOYER P., ZORN J. R., CEDARD L., 1988. Immunoreactive renin variations as a predictive index of the outcome after in vitro fertilization and embryo transfer or gametes intrafallopian transfer. 4th Ann. Meet. European Soc. Human Reprod. Barcelone. July 1988 
(13) ZORN J. R., BOYER P., GUICHARD A., 1987. Never on Sunday : programming for IVF-ET and GIFT. Lancet (1), 385-386.

(14) MENARD J., GUYENNE T., CORVOL D., PAU B., SIMON D., RONCUCCI R., 1985. Direct immunometric assay of active renin in human plasma. J. Hypertension, 3 (suppl. 3), S275-S277.

(15) NAVOT D., MARGALIOTH E. J., LAUFER N., BIRKENFELD A., RELOU ROSLER A., SHENKER J. G., 1987. Direct correlation between plasma renin activity and severity of the ovarian hyperstimulation syndrome. Fert. Steril., 48, 57-61.

(16) DERKX F. H. M., ALBERDA A. T., DE JONG F. H., ZEILMAKER F. H., MAKOVITZ J.W., SCHALEKAM M. A. D. H., 1987. Source of plasma prorenin in early and late pregnancy: observations in a patiente with primary ovarian failure. J. clin. Endocr. Metab., 65, 349-354.

(17) SYMONDS E. M., 1988. Renin and reproduction. Am. J. Obstet. Gynec., 158, 754-761.

(18) DO Y. S., SHERROD A., LOBO R. A., PAULSON R. J., SHINAGAWA T., CHEN S., KJOS S., HSUEH W. A., 1988. Human ovarian theca cells are a source of renin. Proc. nat. Acad. Sci. USA, 85, 1957-1961

(19) DERKX F. H. M., 1987. Human prorenin. Thesis ICG Printing Dordrecht. 